ENSINO E PESQUISA EM ADMINISTRAÇÃO 


\title{
RESISTÊNCIA AO USO DO M-LEARNING POR PROFESSORES DO ENSINO SUPERIOR
}

\author{
RESISTANCE TO THE USE OF M-LEARNING BY HIGHER \\ EDUCATION TEACHERS
}

Renata Kurtz

Universidade estadual do Rio de Janeiro

Jorge Brantes Ferreira

Pontifícia Universidade Católica do Rio de Janeiro

Angilberto Sabino de Freitas

Universidade Unigranrio
Jorge Ferreira da Silva

Pontifícia Universidade Católica do Rio de Janeiro

Data de submissão: 29 set. 2018. Data de aprovação:

10 jan. 2019. Sistema de avaliação: Double blind review.

Universidade FUMEC / FACE. Prof. Dr. Henrique Cordeiro

Martins. Prof. Dr. Cid Gonçalves Filho.

\section{RESUMO}

Objetivo deste artigo é investigar os efeitos da resistência ao M-learning sobre a atitude e intenção de adoção desta inovação de ensino no educação superior, sob o ponto de vista de professores universitários. Para tal, foi realizada uma pesquisa com professores do ensino superior público e/ou privado no Brasil, em que se obteve 512 respostas válidas. $O$ modelo foi estimado com a técnica de modelagem de equações estruturais. Os resultados sugerem que a resistência dos professores ao M-learning seja antecedente da atitude geral de adoção ao M-learning e que os professores percebem que seu desempenho profissional pode melhorar com o uso do M-learning. Os resultados representam avanços no conhecimento sobre adoção do M-learning na perspectiva dos professores, pouco explorada pela literatura e sugerem importantes ações gerenciais para instituições de ensino superior acerca da adoção e implementação de inovações.

PALAVRAS-CHAVE

Mobile Learning. Adoção de inovações. Resistência, Professores. Atitude. 


\section{ABSTRACT}

The goal of this study is to investigate the effects that professors' resistance to $m$-learning has on their attitude and their adoption of this teaching innovation in higher education. To achieve this, a survey with higher education instructors from public and private institutions in Brazil was conducted. With $5 / 2$ valid responses, the proposed relationships, linking innovation attributes to resistance, attitude and adoption intention, were estimated via structural equation modeling. The results suggest that professors' resistance to m-learning is an important antecedent to their general attitude towards m-learning, with instructors perceiving that their teaching perfomance can be improved with through m-learning usage. The study's results and discussion represent a relevant contribution on the understanding of m-learning adoption by higher education professionals, a group of stakeholders seldom evaluated in m-learning research. Finally, the study concludes with suggestions to higher education institutions on how to foster the adoption and successful implementation of m-learning.

\section{KEYWORDS}

Mobile Learning. Innovation adoption. Resistance. Teachers. Attitude

\section{INTRODUÇÃO}

Apesar do crescente uso dos dispositivos móveis, como smartphones, celulares e tablets, o seu uso na educação ainda é um desafio para os educadores, agentes essenciais ao lado das instituições de ensino para a implementação bem sucedida do Mobile-learning (ou M-learning) (KHADDAGE; CHRISTENSEN; LAI et al., 20I5). Apesar dos dispositivos móveis serem amplamente adotados por alunos, professores e pela população em geral, o M-learning parece ainda não ser ter sido adotado pelos professores (KHADDAGE; CHRISTENSEN; LAl et al., 20 I 5).A integração do M-learning ao trabalho docente pode significar uma mudança de paradigma para os professores e, embora reconheçam a tendência crescente de seu uso na educação presencial ou a distância, eles podem não se mostrar inclinados a utilizá-lo em seu trabalho, demonstrando resistência a essa modalidade de ensino (KURTZ ; PINA; FERREIRA et al., 20I4; PINA; KURTZ; FREITAS et al., 20I5).

O M-learning pode ser descrito como uma modalidade de ensino que, por meio de redes sem fio, utiliza dispositivos móveis para facilitar: (i) a transmissão de informações; (ii) o acesso a conteúdos; (iii) a interação entre alunos e professores; e (iv) a consulta e compartilhamento de materiais diversos em qualquer lugar e a qualquer momento (MOTIWALLA, 2007; FERREIRA; KLEIN; FREITAS et al., 20I3). A essas características, pode-se acrescentar a convergência de várias mídias e funções em um aparelho único (JENKINS, 2008). Por fim, Churchill e Churchill (2008) indicam o seu compartilhamento com outras pessoas, propiciado pela conectividade social 
para a aprendizagem.

Tão importante quanto o papel do professor no processo de adoção do M-learning, é entender como o professor reage a essa inovação. Ao pesquisar a literatura, percebe-se que a adoção do M-learning por professores é ainda pouco explorada, sendo apenas recentemente tema de pesquisas (SÁNCHEZ-PRIETO; OLMOS-MIGUELÁÑEZ; GARCÍA-PEÑALVO, 2016 ; AL-EMRAN; ELSHERIF; SHAALAN, 20I6).

Nesse contexto, 0 entendimento da perspectiva docente e de seu papel no M-learning parece ser essencial para a sua eficácia tanto no que se refere à própria compreensão do M-learning pelos professores para que possam se engajar a ele quanto para o desenvolvimento de ações institucionais de capacitação docente para esta modalidade de ensino (HASHIM;TAN; RASHID, 20I5).

Dessa forma, o objetivo deste estudo é identificar os fatores que influenciam a resistência à intenção de adoção do M-learning por professores no ensino superior, perspectiva ainda pouco explorada e fragmentada no estudo da adoção de inovações.

\section{REVISÃO DE LITERATURA Adoção de Inovações}

$\bigcirc$ avanço das tecnologias móveis e digitais representa uma inovação e um desafio a docentes e Instituições de Ensino Superior (IES) para se adequarem a novos métodos e práticas educativas que incorporem o seu uso (HASHIM;TAN; RASHID, 20I5). Apesar das evidências de que docentes têm papel essencial no uso do M-learning (HWANG; WU, 20I4), o uso do telefone celular em si tem sido muitas vezes proibido no ambiente educativo pelo próprio docente (KHADDAGE; CHRISTENSEN; LAl et al., 20I5).

Para investigar a adoção de inovações de novos produtos e serviços, diversos modelos e teorias têm sido propostos. Dentre esses modelos, pode-se citar a teoria da difusão de inovações (IDT - ROGERS, 2003), a teoria da ação racionalizada (TRA) (FISHBEIN; AJZEN, I975), a teoria do comportamento planejado (TPB) (AJZEN, 199I) e as teorias que estudam especificamente as inovações tecnológicas, como o modelo de aceitação da tecnologia (TAM) (DAVIS, I989; DAVIS; BAGOZZI; WARSHAW, 1989), a teoria unificada de aceitação e uso da tecnologia (UTAUT) (VENKATESH; MORRIS; DAVIS et al., 2003) e, para o caso específico do M-learning, o modelo de aceitação do Mobile Learning (MLAM) (AKOUR, 20I0).

Dentre elas, a IDT é considerada uma das mais adequadas ao estudo das inovações em virtude da fundamentação que oferece para explicar o que impacta na adoção de inovações em amplos contextos (trabalho, educação, vida doméstica, consumo etc.). Adicionalmente, a IDT é considerada mais apropriada ao presente estudo pelo fato de o M-learning ainda não ter sido largamente adotado, o que pode prejudicar a investigação da percepção de uso efetivo; e pela similaridade constatada entre os construtos das teorias citadas e a consequente possibilidade de equivalência entre eles, conforme apontada no quadro I.

Particularmente, no que diz respeito à avaliação da adoção de tecnologias móveis, a IDT tem sido aplicada em pesquisas que estudam fatores que afetam a adoção de inovações por indivíduos e organizações (DUAN; HE; FENG et al., 20I0; 
QUADRO 1 - Comparação e equivalência entre os construtos

\begin{tabular}{|c|c|c|c|c|c|}
\hline $\begin{array}{l}\text { IDT } \\
\text { (Rogers, 2003, Duan et } \\
\text { al, 2010) }\end{array}$ & $\begin{array}{c}\text { TRA } \\
\text { (Fishbein; } \\
\text { Ajzen, 1975) }\end{array}$ & $\begin{array}{c}\text { TPB } \\
\text { (Ajzen, 1985) }\end{array}$ & $\begin{array}{l}\text { TAM (Davis et } \\
\text { al., 1989) }\end{array}$ & $\begin{array}{c}\text { UTAUT } \\
\text { (Venkatesh } \\
\text { et al., 2003) }\end{array}$ & $\begin{array}{c}\text { MLAM } \\
\text { (Akour, 2010) }\end{array}$ \\
\hline Vantagem relativa & $\begin{array}{c}\text { Sem } \\
\text { equivalência }\end{array}$ & $\begin{array}{c}\text { Sem } \\
\text { equivalência }\end{array}$ & $\begin{array}{l}\text { Utilidade } \\
\text { percebida }\end{array}$ & $\begin{array}{l}\text { Expectativa de } \\
\text { desempenho }\end{array}$ & Utilidade \\
\hline Complexidade & $\begin{array}{c}\text { Sem } \\
\text { equivalência }\end{array}$ & $\begin{array}{c}\text { Sem } \\
\text { equivalência }\end{array}$ & $\begin{array}{c}\text { Facilidade de uso } \\
\text { percebida }\end{array}$ & $\begin{array}{c}\text { Expectativa de } \\
\text { esforço }\end{array}$ & Facilidade de uso \\
\hline Compatibilidade & $\begin{array}{c}\text { Sem } \\
\text { equivalência }\end{array}$ & $\begin{array}{l}\text { Controle do } \\
\text { comportamento } \\
\text { percebido }\end{array}$ & $\begin{array}{c}\text { Sem } \\
\text { equivalência }\end{array}$ & $\begin{array}{l}\text { Condições } \\
\text { facilitadoras }\end{array}$ & $\begin{array}{c}\text { Compromisso da } \\
\text { universidade } \\
\text { (antecedente de } \\
\text { utilidade e facilidade) }\end{array}$ \\
\hline Experimentabilidade & $\begin{array}{c}\text { Sem } \\
\text { equivalência }\end{array}$ & $\begin{array}{c}\text { Sem } \\
\text { equivalência }\end{array}$ & $\begin{array}{c}\text { Sem } \\
\text { equivalência }\end{array}$ & $\begin{array}{c}\text { Sem } \\
\text { equivalência }\end{array}$ & Sem equivalência \\
\hline Observabilidade & $\begin{array}{c}\text { Sem } \\
\text { equivalência }\end{array}$ & $\begin{array}{c}\text { Sem } \\
\text { equivalência }\end{array}$ & $\begin{array}{c}\text { Sem } \\
\text { equivalência }\end{array}$ & $\begin{array}{c}\text { Sem } \\
\text { equivalência }\end{array}$ & Sem equivalência \\
\hline Sem equivalência & $\begin{array}{l}\text { Normas } \\
\text { subjetivas }\end{array}$ & $\begin{array}{l}\text { Normas } \\
\text { subjetivas }\end{array}$ & $\begin{array}{c}\text { Sem } \\
\text { equivalência }\end{array}$ & Influência social & $\begin{array}{l}\text { Influência extrínseca } \\
\text { (antecedente de } \\
\text { utilidade e facilidade) }\end{array}$ \\
\hline Sem equivalência & $\begin{array}{l}\text { Sem } \\
\text { equivalência }\end{array}$ & $\begin{array}{c}\text { Sem } \\
\text { equivalência }\end{array}$ & $\begin{array}{c}\text { Sem } \\
\text { equivalência }\end{array}$ & $\begin{array}{c}\text { Sem } \\
\text { equivalência }\end{array}$ & $\begin{array}{l}\text { Prontidão do estudante } \\
\text { (antecedente de } \\
\text { utilidade e facilidade) }\end{array}$ \\
\hline Sem equivalência & $\begin{array}{c}\text { Sem } \\
\text { equivalência }\end{array}$ & $\begin{array}{c}\text { Sem } \\
\text { equivalência }\end{array}$ & $\begin{array}{l}\text { Sem } \\
\text { equivalência }\end{array}$ & $\begin{array}{c}\text { Sem } \\
\text { equivalência }\end{array}$ & $\begin{array}{l}\text { Qualidade do serviço } \\
\text { (antecedente de } \\
\text { utilidade e facilidade) }\end{array}$ \\
\hline
\end{tabular}

MACHADO; BELLINIC; LEITE, 20 I 2; MA; LEE; GOH, 20I4; KAPOOR; DWIVEDI; WILLIAMS, 20I4; KURTZ; MACEDOSOARES; FERREIRA et al., 20I5; PINA; KURTZ; FREITAS et al., 20I5; CHIYANGWA;ALEXANDER, 2016).

De acordo com a IDT, no processo decisório de aceitação da tecnologia, a atitude é influenciada pela forma como o indivíduo percebe um conjunto de atributos inerentes à inovação. Esses atributos são: a vantagem relativa, que é o grau com que uma inovação é percebida como melhor do que a ideia precursora, ou seja, como sendo uma melhoria em relação ao desempenho obtido pela forma de trabalho anteriormente exercida; a compatibilidade, que é o grau com que uma inovação é percebida como sendo consistente e coerente com os valores existentes, as expe- riências e as necessidades dos potenciais adotantes; a complexidade, que é o grau com que uma inovação é percebida como difícil de entender e utilizar por seus potenciais adotantes; a "experimentabilidade" [nome nosso], que é o grau com que uma inovação pode ser minimamente experimentada por seus potenciais adotantes antes da decisão de adoção e; a "observabilidade" [nome nosso], que é o grau com que os resultados de uma inovação são perceptíveis para os outros e para potenciais adotantes (ROGERS, 2003).

Kurtz, Pina, Ferreira et al. (2014) e Pina, Kurtz, Freitas et al. (20I5) investigaram qualitativamente as percepções de docentes da educação superior sobre a adoção do M-learning, com base nos atributos da inovação de Rogers (2003) e identificaram que os fatores que pareceram facilitar a adoção 
foram a vantagem relativa e a compatibilidade. Já a complexidade foi identificada como fator que possivelmente dificulta a adoção do M-learning. Não surgiram referências aos atributos "experimentabilidade" e "observabilidade" no estudo. Além dos construtos da IDT pesquisados, três novas categorias emergiram como desvantagens do M-learning, barreiras ao M-learning e necessidades para a adoção de M-learning.

Por fim, Khaddage, Christensen, Lai et al. (20I5) apontam que a falta de habilidades técnicas e pedagógicas, a falta de teoria pedagógica e de evidências da eficácia do M-learning e a pouca orientação para o uso e a falta de suporte técnico podem levar à resistência a sua adoção por parte dos professores. Diante disso, se mostrou necessário estudar o construto resistência para investigação de sua influência na atitude de adoção do M-learning pelos professores.

\section{RESISTÊNCIA}

A meta-análise de Lapointe e Rivard (2005) sobre resistência à implementação de tecnologia da informação (TI) revela que os trabalhos que enfocam a resistência à inovação são raros e fragmentados, indicando que o tema precisa ser meIhor pesquisado. Estudos mais recentes sobre adoção de inovações como os de Sanford e Oh (2010), Heidenreich e Kraemer (20I5) e Sánchez-Prieto, Olmos-Migueláñez e García-Peñalvo (2016) confirmam essa afirmação.

Zaltman e Duncan (1977) definem resistência à mudança como qualquer conduta para manter o status quo diante da pressão para alterá-lo, ressaltando que ela pode não ser sinônimo de falta de aceitação ou o oposto da aceitação. Em outras palavras, como a mudança envolve alterações no sta- tus quo, é provável que a resistência ocorra quando qualquer mudança acontecer.

No que se refere à resistência à tecnologia, Orlikowski e Gash (1994) apontam que as reações dos indivíduos para a introdução de tecnologias de informação dependem de sua interpretação da tecnologia. Para interagir com a tecnologia, os indivíduos precisam dar sentido a ela, e nesse processo desenvolvem premissas particulares, expectativas e certo conhecimento sobre ela, que contribuirão para formar as ações subsequentes acerca do uso da tecnologia, o que chamaram de estruturas tecnológicas. Tais estruturas são úteis, pois facilitam a interpretação de situações ambíguas, reduzindo incertezas em condições de mudança e proporcionam a base para a ação. Por outro lado, são restritivas ao reforçar crenças em premissas e conhecimentos sem a devida reflexão, ao distorcer informações a fim de adequá-las às estruturas cognitivas já existentes e ao inibir processos criativos de solução de problemas, impedindo os indivíduos de formar novas estruturas.

De forma geral, o construto resistência tem sido tema de pesquisas na literatura na implementação de novos sistemas. Joshi (I99|) investiga a resistência à mudança com base na teoria da equidade, em que o indivíduo avalia a mudança em seu status com o novo sistema e o compara ao estado corrente da organização e dos demais membros de seu grupo de referência na organização. Marakas e Hornik (1996) explicam a resistência como resposta passiva-agressiva a ameaças e estresses que o indivíduo associa às incertezas provocadas pelo novo sistema. Martinko, Henry e Zmud (1996) propõem um modelo de atribuição causal das reações dos indivíduos à implementação de TI (considerando 
as características da nova tecnologia, fatores internos e externos e experiências de sucesso e fracasso deles com tecnologias semelhantes) que influenciam suas expectativas sobre a eficácia e os resultados de desempenho com o novo sistema. Lapointe e Rivard (2005) avaliaram a resistência de grupos de médicos à implementação de prontuários eletrônicos em hospitais e observaram adoção, neutralidade, apatia, resistência passiva, resistência ativa e resistência agressiva.

Já os trabalhos de Ellen, Bearden e Sharma (199I), Norzaidi, Chong e Salwani (2008) e Sanford e Oh (20l0) representam importantes contribuições ao estudo da resistência. Ellen, Bearden e Sharma (I99I) definiram a resistência à mudança como uma resposta avaliativa para manter o status quo, ou seja, gerado por forças incentivadoras da manutenção do comportamento existente. Norzaidi. Chong e Salwani (2008) investigaram a influência do ajuste tarefa-tecnologia da resistência percebida, da resistência do usuário e do uso da tecnologia para estudar o desempenho profissional de gerentes. Por fim, Sanford e Oh (2010) pesquisaram os efeitos da resistência ao uso da tecnologia móvel no trabalho em uma agência governamental do setor imobiliário. Seus resultados mostram que a resistência é um possível antecedente ao uso, uma vez que a resistência antecede a mudança. Adicionalmente, enquanto o uso da $\mathrm{TI}$ caracteriza-se como um comportamento, a resistência não é um comportamento, mas uma força cognitiva, como uma intenção na tentativa de preservar o status quo, o que tem um impacto negativo na mudança.

Para esta pesquisa, adotou-se a definição de resistência à mudança de Sanford e Oh
(2010), que a define como uma força não específica a um sistema, uma tentativa de manter o status quo na sua prática profissional, o que inspirou a primeira questão de pesquisa deste artigo: quais são os fatores que geram a resistência dos professores ao M-learning?

Pelo fato de a definição adotada enfocar uma abordagem ampla dos fatores determinantes que levariam o indivíduo a tentar manter o status quo de seu comportamento no trabalho, ao invés de investigar somente características específicas do sistema inovador, os atributos da teoria de difusão de inovações de Rogers (2003) parecem ser os mais apropriados para a investigação de tais antecedentes, o que resultou nas seguintes hipóteses:

HI:Vantagem relativa terá efeito direto e negativo sobre a resistência dos professores ao M-learning.

H2: Complexidade terá efeito direto e positivo sobre a resistência dos professores ao M-learning.

H3: Compatibilidade terá efeito direto e negativo sobre a resistência dos professores ao M-learning.

H4: "Experimentabilidade" terá efeito direto e negativo sobre a resistência dos professores ao M-learning.

H5: "Observabilidade" terá efeito direto e negativo sobre a resistência dos professores ao M-learning.

Suporte Institucional

Diversos trabalhos (IGBARIA, I990; MOORE; BENBASAT, I99I; IGBARIA, 1993; AKOUR, 2010) demonstram que o apoio institucional pode reunir condições organizacionais que facilitam a adoção de sistemas de inovação.Akour (2010) adotou o construto comprometimento da universidade, igualando-o ao suporte da universi- 
dade com base nas dimensões treinamento e suporte ao usuário para o estudo do M-learning no Ensino Superior. Além do mais, com base nos resultados de Kurtz, Pina, Ferreira et al. (20/4), Pina, Kurtz, Freitas et al. (20I5) e Khaddage, Christense, Lai et al. (20I5), esse construto foi acrescido ao modelo da presente pesquisa como suporte institucional com o objetivo de se investigar sua influência na resistência dos docentes ao M-learning. Assim é proposta a sexta hipótese:

H6: Suporte institucional terá efeito direto e negativo sobre a resistência dos professores ao M-learning.

Com base no que foi apresentado, julgouse relevante investigar a influência da resistência dos professores ao M-learning na atitude geral formada no processo de decisão de adoção da inovação; e também a influência da atitude geral dos professores de adoção do M-learning na sua intenção de adoção do M-learning em seu trabalho, resultando nas hipóteses finais do modelo (Figura I):
H7: Resistência dos professores ao M-learning terá efeito negativo sobre a atitude geral de adoção dos professores ao M-learning.

H8: A atitude geral dos professores de adoção do M-learning terá efeito positivo sobre a intenção de adoção dos professores ao M-learning.

\section{METODOLOGIA}

Com o objetivo de realizar o teste das hipóteses propostas para o estudo, foi realizada uma cross-sectional survey (PARASURAMAN; GREWAL; KRISHNAN, 2006) com uma amostra não probabilística da população de interesse. Grande parte dos estudos sobre aceitação de tecnologia, como o e-learning e o M-learning, faz uso desta mesma forma de pesquisa (ONG; LAI, 2006).

\section{Operacionalização das variáveis}

O estudo utilizou escalas anteriormente criadas e validadas pela literatura para a mensuração de todos os construtos do modelo de pesquisa. Os itens foram tradu-

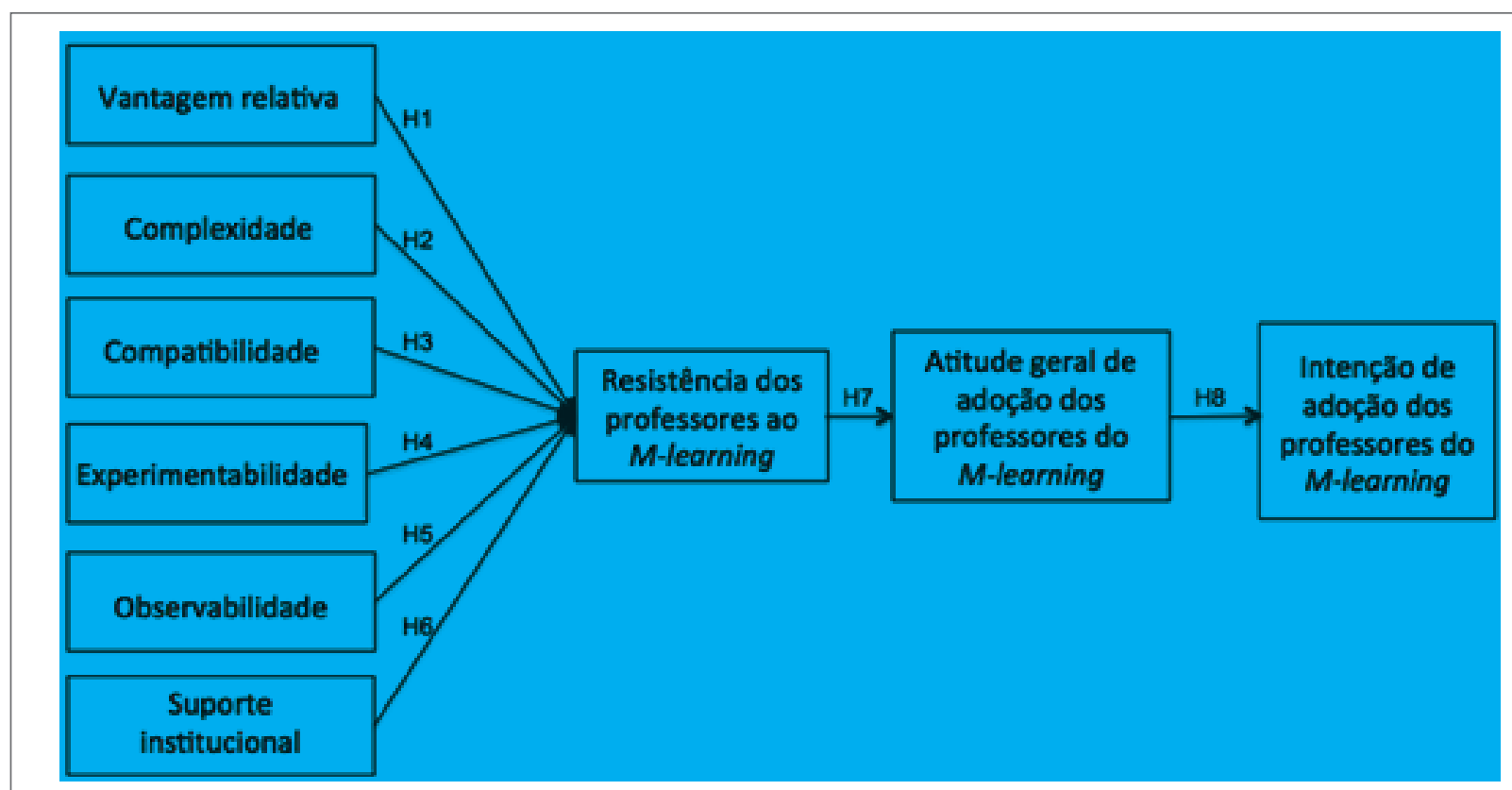

FIGURA 1 - Modelo proposto e hipóteses da pesquisa.

Fonte: Elaborado pelos autores 
zidos do inglês para o português por um tradutor, e a tradução foi avaliada por três pesquisadores especialistas na área, conforme recomenda Sperber (2004), a fim de garantir a validade de face dos construtos mensurados. Em seguida, foi realizada a retradução (back translation) para o inglês, por uma especialista que não trabalhou na primeira tradução.

Foram adaptadas escalas de Duan, He, Feng et al. (20 I0),Akour (20 I0), Kurtz, Pina, Ferreira et al.. (20l4) - essas, validadas em Taylor e Todd (1995) e Lu, Zhou e Wang (2009) - e Sanford e Oh (20 I 0), resultando nos itens apresentados a seguir:

Vantagem relativa percebida: escala de Duan, He, Feng et al. (20 I0), com 7 itens;

Compatibilidade percebida: escala de Duan, He, Feng et al. (20 I0), com 5 itens;

Complexidade percebida: escala de Duan, He, Feng et al. (20 I0), com 6 itens;

Experimentabilidade percebida: escala de Duan, He, Feng et al. (20 I0), com 4 itens; Observabilidade percebida: escala de Duan, He, Feng et al. (20 I0), com 5 itens;

Suporte institucional: escala de Akour (20I0), com 4 itens;

Resistência à mudança: escala de Sanford e Oh (2010), com 4 itens;

Atitude com relação à adoção: escalas de Taylor e Todd (1995) e de Lu, Zhou e Wang, (2009), com 3 itens;

Intenção de uso: escala de Sanford e Oh (2010), com 3 itens.

Deste modo, o instrumento de pesquisa contou com um total de 4 I itens, acrescidos de oito relativos ao perfil demográfico e profissional dos respondentes, quatro itens relativos à percepção do M-learning e seis itens referentes ao perfil de uso da tecnologia móvel.

pré-teste foi realizado em duas eta- pas: o pré-teste dos itens propriamente ditos e o pré-teste da veiculação e aplicação on-line do questionário. Foram feitos os ajustes necessários para a compreensão correta das questões. No segundo teste, os respondentes revelaram mais facilidade para compreender o conceito sobre M-learning na versão ajustada.

\section{Amostra e Coleta de dados}

O questionário foi enviado a professores por meio eletrônico de três formas: (i) diretamente aos e-mails divulgados na internet pelas IES com programas associados à ANPAD; (ii) remetido respectivamente a listas de discussão por e-mail formadas por professores universitários; (iii) enviado a grupos de professores universitários na rede social Facebook. Não foi feita distinção entre professores da modalidade de ensino presencial e on-line.

A amostra totalizou 549 respostas, das quais 37 foram eliminadas por serem de professores que não atuam no ensino superior. Não houve dados ausentes. A amostra final, contendo somente professores do ensino superior, foi formada por 512 questionários válidos.

No que se refere aos dados demográficos, dos 512 professores, 54, I\% eram do sexo masculino. A respeito da idade, 24,6 $\%$ se enquadram na faixa de 31 a 40 anos; $28,1 \%$ com 4 I a 50 anos; e $27,7 \%$ com 5 I a 60 anos. A idade média verificada foi de 47,6 anos. Do total, 50\% trabalham em IES privadas, $42,6 \%$ trabalham em IES públicas e 7,4\% trabalham nas duas, privada e pública. A maior parte dos professores (53,5\%) atua no ensino superior há mais de 10 anos; $22,9 \%$ atuam no ensino superior entre 5 e 10 anos; e os demais $23,6 \%$ são professores universitários há menos de 5 anos. 
Quando perguntado a respeito das tecnologias usadas em sala de aula, as respostas se concentraram inicialmente no uso do Power Point (486 professores), seguidas de acesso à internet em aula (35I professores), uso de softwares específicos (243 professores) e plataformas on-line da universidade como apoio ao ensino presencial (246 professores). Os resultados mostraram também que 258 respondentes já trabalharam com educação on-line, 26 relataram outros usos e dois professores não usam o computador em aula.

No que concerne especificamente ao uso de tecnologia móvel, $99 \%$ possuem dispositivo móvel de qualquer tipo e somente I\% não possui. A respeito do tempo de uso diário da internet pelo dispositivo móvel, o maior grupo, formado por $34,4 \%$, relatou usá-la menos de 2 horas por dia; $23 \%$ afirmaram usá-la entre 2 e 3 horas por dia; 17,6\% relataram utilizá-la entre 3 e 4 horas diárias; e 22,3\% declararam usá-la por mais de 4 horas por dia.

Diante do uso pessoal do dispositivo móvel, $92,2 \%$ o utilizam para fazer e receber ligações telefônicas; $92 \%$ para enviar e receber mensagens de texto; $90 \%$ para acessar a internet; $86,3 \%$ para fotografar ou filmar; 85,5\% para ler e escrever e-mails; $77 \%$ para usar a calculadora; 74,6\% para usar aplicativos dos dispositivos móveis; 69,3\% para assistir a vídeos; 66,8\% para enviar e receber mensagens de voz; 67,8\% para interagir com outros profissionais do trabalho; e $66 \%$ para compromissos em calendário ou agenda. Em menor número, também surgiu a leitura de livros eletrônicos $(40,4 \%)$ e somente $3,9 \%$ para interagir com alunos.

\section{RESULTADOS}

\section{Teste para Viés de Método Comum}

Dado que neste estudo tanto as variá- veis independentes quanto as dependentes medem opiniões dos mesmos informantes, a variância de método comum poderia representar um problema. Seguindo sugestão de Podsakoff e Organ (1986), o teste de um fator de Harman foi empregado para examinar se algum viés de método comum estava presente nos dados. $O$ resultado da análise de componentes principais indicou a presença de sete fatores com autovalor maior do que I, com nenhum desses fatores capturando uma parte exagerada da variância total (o fator mais explicativo correspondia a $37,78 \%$ da variância total dos dados). Sendo assim, acredita-se que não houve problemas de viés de método comum neste estudo.

\section{Modelo de mensuração}

O modelo de mensuração foi inicialmente testado com todos os $4 \mathrm{I}$ itens. A análise da matriz de covariância dos resíduos padronizados da análise fatorial confirmatória apontou itens que poderiam influenciar o ajuste do modelo, e o modelo foi refinado, eliminando-se sete itens que não se mostraram adequados com a estrutura dos respectivos construtos propostos. $O$ modelo de mensuração final contou, então, com 34 itens dos $4 \mathrm{I}$ iniciais. Assim, o modelo de mensuração final apresentou melhores índices de ajuste com: RMSEA de 0,07 (com C.I. de 0,066 a 0,074); CFI de 0,93; IFI de 0,93; TLI de 0,92; e c2 $=\mid 720,542$, d.f. $=49|, p<0,00|$, c2 /d.f. $=3,50)$, mostrando-se mais apropriado do que o modelo inicial, sugerindo um ajuste satisfatório dos dados ao modelo de pesquisa (HU; BENTLER, 1999).

A validade de face foi garantida pela escoIha criteriosa de escalas já utilizadas e validadas na literatura; pelo processo de tradução 
e de avaliação por especialistas em língua inglesa e no assunto, respectivamente; e pela realização do pré-teste em duas etapas com amostras da população de interesse.

A validade nomológica foi examinada pela avaliação da matriz de correlação entre os construtos para verificar se as relações entre os construtos correspondem ao preconizado pela teoria. As correlações (Complexidade/Vantagem Relativa; Complexidade/ Experimentabilidade; Complexidade/Observabilidade; Complexidade/Suporte institucional; Complexidade/Atitude geral de adoção dos professores ao M-learning; Complexidade/Intenção de adoção dos professores ao M-learning) não se mostraram significativas a um nível de significância de 0.005. Adicionalmente, Experimentabilidade/Resistência dos professores ao M-learning e Observabilidade/Resistência dos professores ao M-learning não apresentaram correlação significativa. Todas as demais correlações se mostraram significativas e com as relações (positivas ou negativas) esperadas. Assim, os construtos apresentaram validade nomológica.

Para análise da consistência interna e da confiabilidade das escalas, todos apresentaram valores acima de 0,7 tanto para ao coeficiente alpha de Cronbach quanto para a confiabilidade composta (CR) estando de acordo com a literatura (LUND, 200I). Os valores de CR variaram entre 0,83 e 0,95, atendendo aos padrões requeridos. $A$ respeito da validade convergente, a variância extraída média (AVE) para cada construto correspondeu ao mínimo de 0,50, mínimo recomendado como adequado (FORNELL; LARCKER, 198I), com todos os valores entre 0,56 e 0,83 , revelando validade convergente das escalas adotadas.

Para a verificação da validade discriminante, comparou-se a variância extraída média de cada construto com a variância compartilhada entre todos os pares de construtos. Quase todas as variâncias compartilhadas foram inferiores à variância extraída média para cada construto, com exceção apenas de intenção de adoção dos professores ao M-learning, que apresentou AVE inferior à variância compartilhada com o construto atitude geral em relação à adoção do M-learning. Pode-se sugerir que os respondentes podem ter sentido certa dificuldade de distinguir suas respostas sobre intenção de adoção dos professores ao M-learning, em comparação com suas respostas sobre atitude geral de adoção dos professores ao M-learning pelo fato da inovação ainda não ser bem conhecida pelos respondentes.

\section{MODELO ESTRUTURAL}

A avaliação das relações entre os construtos foi realizada por meio da modelagem de equações estruturais. A razão $\chi 2$ /d.f. foi 6,445 , superior ao 3,0 sugerido por Byrne (2010). Já os índices incrementais foram próximos de 0,9, embora não tenham superado esse valor, conforme recomendado pela literatura, Hinkin (1995) admitiu que valores próximos de 0,85 são aceitáveis. 0 CFI obtido foi de 0,83 , o TLI foi de 0,817 e o IFI foi de 0,83I. Os índices de ajuste absoluto obtidos mostraram valores acima do limite de 0,08, conforme recomendado por Hu e Bentler (1999) e Byrne (20 I0). O RMSEA foi de 0, 103 (C.I. de 0, 100 a 0, I07) e o SRMR foi de 0,3I73. Apesar de o conjunto de índices apresentado sugerir que o modelo estrutural forneceu um ajuste periférico, os índices incrementais apresentaram valores próximos aos considerados ideais de forma que se decidiu prosseguir com o teste das hipóteses de pesquisa. 
TABELA 1 - Hipóteses, coeficientes padronizados estimados e significâncias para o modelo estrutural

\begin{tabular}{l|c|c|c}
\hline Relação Proposta & Coeficiente Padronizado & p-value & Hipótese Verificada \\
\hline H1: ADV $\rightarrow$ RES & $-0,163$ & $<0,001$ & $\operatorname{sim}$ \\
\hline H2: CLEX $\rightarrow$ RES & 0,214 & $<0,001$ & $\operatorname{sim}$ \\
\hline H3: COMP $\rightarrow$ RES & $-0,133$ & 0,002 & $\operatorname{sim}$ \\
\hline H4: TRIAL $\rightarrow$ RES & $-0,014$ & 0,748 & não \\
\hline H5: OBSR $\rightarrow$ RES & 0,246 & $<0,001$ & não \\
\hline H6: US $\rightarrow$ RES & $-0,167$ & $<0,001$ & $\operatorname{sim}$ \\
\hline H7: RES $\rightarrow$ ATT & $-0,36$ & $<0,001$ & $\operatorname{sim}$ \\
\hline H8: ATT $\rightarrow$ INT & 0,909 & $<0,001$ & $\operatorname{sim}$ \\
\hline
\end{tabular}

Fonte: Elaborado pelos autores.

O teste de hipóteses (Tabela I) foi conduzido por meio da verificação de magnitude, direção e significância dos coeficientes padronizados estimados para as relações do modelo estrutural. O modelo foi capaz de explicar 17,9\% da variância da resistência dos professores ao M-learning, 13\% da variância da atitude geral de adoção dos professores ao M-learning e $82,7 \%$ da variância da intenção de adoção dos professores ao M-learning.

\section{Comparação com o modelo alternativo}

Conforme a recomendação de Anderson e Gerbing (1988), foi feita uma comparação entre o modelo proposto e modelos alternativos para avaliar a força e a relevância de diferentes relações entre os construtos.

Hair, Black, Babin et al. (2009) afirmam que, em teoria, um construto mediador facilita a relação entre os outros construtos envolvidos e se o modelo com mediação fornece um bom ajuste, o papel mediador é igualmente sustentado.As alterações propostas no modelo alternativo são baseadas na inclusão de efeitos diretos entre os construtos antecedentes (vantagem relativa, complexidade, compatibilidade, experimentabilidade, observabilidade e suporte institucional) na atitude geral de adoção dos professores ao
M-learning. Dessa forma, o efeito da mediação desses construtos antecedentes sobre a atitude geral de adoção dos professores ao M-learning poderá ser comparado pelas relações diretas, sem mediação.

O modelo alternativo (figura 2) forneceu melhores ajustes do que o modelo original em todos os índices (incrementais, absoluto e RMSEA).A razão $\chi 2$ /d.f. obtida foi de 5, 164 e os índices incrementais demonstraram valores mais próximos a 0,9 (CFI de 0,87,TLI de 0,86 e IFI de 0,87$)$. O RMSEA e o SRMR também tiveram valores mais baixos $(0,09 \mathrm{e}$ 0,244 respectivamente).

A tabela 2 apresenta os coeficientes padronizados de cada relação estimada no modelo alternativo, com as respectivas significâncias.

O exame do R2 para os construtos endógenos mostrou que os valores da proporção da variância explicada da resistência dos professores ao M-learning foram quase os mesmos ( $17,9 \%$ no modelo original e 17,3\% no modelo alternativo). A proporção da variância observada da intenção de adoção dos professores ao M-learning foi de $82,7 \%$ no modelo original e de $75,3 \%$ no modelo alternativo. Entretanto, o modelo alternativo foi capaz de explicar melhor a atitude geral de adoção dos professores ao M-learning com proporção da variância explicada de $69,8 \%$, contra I3\% da propor- 




\section{FIGURA 2 - Modelo alternativo}

Fonte: Elaborado pelos autores.

TABELA 2 - Hipóteses, coeficientes padronizados estimados e significâncias para o modelo alternativo

\begin{tabular}{|c|c|c|c|}
\hline Relação Proposta & Coeficiente Padronizado & p-value & Hipótese Verificada \\
\hline $\mathrm{H} 1: \mathrm{ADV} \rightarrow \mathrm{RES}$ & $-0,161$ & $<0,001$ & $\operatorname{sim}$ \\
\hline H2: CLEX $\rightarrow$ RES & 0,215 & $<0,001$ & $\operatorname{sim}$ \\
\hline H3: COMP $\rightarrow$ RES & $-0,12$ & 0,005 & $\operatorname{sim}$ \\
\hline H4: TRIAL $\rightarrow$ RES & $-0,014$ & 0,75 & não \\
\hline H5: OBSR $\rightarrow$ RES & 0,247 & $<0,001$ & não \\
\hline H6: US $\rightarrow$ RES & $-0,158$ & $<0,001$ & $\operatorname{sim}$ \\
\hline $\mathrm{H} 7: \mathrm{ADV} \rightarrow \mathrm{ATT}$ & 0,287 & $<0,001$ & $\operatorname{sim}$ \\
\hline H8: CLEX $\rightarrow$ ATT & $-0,018$ & 0,549 & não \\
\hline H9: COMP $\rightarrow$ ATT & 0,499 & $<0,001$ & $\operatorname{sim}$ \\
\hline H10: TRIAL $\rightarrow$ ATT & 0,021 & 0,481 & não \\
\hline H11: OBSR $\rightarrow$ ATT & 0,035 & 0,243 & não \\
\hline H12: US $\rightarrow$ ATT & 0,546 & $<0,001$ & $\operatorname{sim}$ \\
\hline H13: RES $\rightarrow$ ATT & $-0,13$ & $<0,001$ & $\operatorname{sim}$ \\
\hline H14: ATT $\rightarrow$ INT & 0,868 & $<0,001$ & $\operatorname{sim}$ \\
\hline
\end{tabular}

Fonte: Elaborado pelos autores

ção da variância da atitude com relação ao M-learning explicada pelo modelo original.

Os resultados avaliados em conjunto mostram que, além do papel mediador da resistência dos professores ao M-learning na atitude geral com relação ao M-learning, as relações diretas dos construtos exógenos na atitude com relação ao M-learning desempenham importante papel na atitude com relação ao M-learning, o que fortalece a decisão de manter seu acréscimo no novo modelo, bem como fortalece a deci- 
são de que o modelo alternativo apresenta melhorias em relação ao modelo original.

\section{DISCUSSÃO}

Os resultados do estudo mostraram razoável ajuste do modelo alternativo, tomando por base os índices de ajuste incrementais e indicaram esse modelo como o mais adequado. $O$ teste de hipóteses verificou significância estatística de 10 das 14 hipóteses testada, sintetizadas na tabela 3.

As hipóteses verificadas no modelo final sugerem que a resistência dos professores ao M-learning seja antecedente à atitude geral de adoção dos professores ao M-learning.

Dentre os determinantes da resistência, os construtos vantagem relativa, complexidade e compatibilidade se mostraram significativos ( $\mathrm{HI}, \mathrm{H} 2$ e H3), apesar de não apresentarem altas magnitudes. Vantagem relativa teve efeitos negativos na resistência dos professores ao M-learning, o que indica que quanto mais melhorias os professores puderem perceber no desempenho de seu trabalho com o uso do M-learning, menor deverá ser a sua resistência a ele. Da mesma forma, compatibilidade teve efeito negativo na resistência. Isso sugere que quanto mais consistente e coerente for a percepção do M-learning pelos professores com seus valores, suas experiências e necessidades, menor será a resistência ao M-learning. A complexidade teve efeito positivo na resistência, indicando que quanto mais difícil for entender e utilizar o M-learning, maior pode ser a resistência a ele.

Observabilidade também mostrou-se significativamente influente na resistência dos professores ao M-learning (H5), porém no sentido positivo, isto é, oposto ao previsto. Isto indica que quanto mais visível for o M-learning, maior a probabilidade de resistência dos professores a essa modalidade. Como dito anteriormente, uma possível explicação para isso pode ser que as poucas oportunidades que os professores têm para observar os resultados do M-learning na prática de outros professores pode prejudicar sua avaliação desses resultados.

Além destes construtos, suporte institucional também se mostrou antecedente à resistência dos professores ao M-learning $(\mathrm{H} 6)$, no sentido negativo previsto na pesquisa.

As relações diretas verificadas dos antecedentes na atitude foram vantagem relativa, compatibilidade e suporte institucional ( $\mathrm{H} 7, \mathrm{H} 9$ e HI2), todas elas nos sentidos previstos pela pesquisa e de acordo com estudos anteriores (ROGERS, 2003, DUAN; HE; FENG et al., 20 I 0; LEE; HSIEH; HSU 20I I; MACHADO; BELLINIC; LEITE, 20I2). Os três antecedentes apresentaram magnitudes superiores nas relações com atitude geral de adoção dos professores ao M-learning em comparação com seus respectivos efeitos na resistência dos professores ao M-learning, com destaque para os efeitos da compatibilidade e do suporte institucional. Tal resultado sugere que quanto maior forem as associações que os professores fizerem com outros usos de tecnologia no ensino conforme seus padrões de referência, e também quanto maior o comprometimento da instituição de ensino com treinamentos, capacitações e suporte contínuo ao professor para o aprendizado e uso do M-learning, mais positivas poderão ser as suas atitudes de adoção do M-learning.

Não houve efeitos verificados da complexidade $(\mathrm{H} 8)$ e da observabilidade $(\mathrm{HII})$ 
TABELA 3 - Síntese dos resultados dos testes de hipóteses do modelo final da pesquisa

Hipóteses de Pesquisa

Hipóteses relacionando os construtos da teoria da difusão de inovações à resistência dos professores ao M-learning

H1: Vantagem relativa terá efeito direto e negativo sobre a resistência dos professores ao M-learning. $\operatorname{sim}$

H2: Complexidade terá efeito direto e positivo sobre a resistência dos professores ao M-learning. $\operatorname{sim}$

H3: Compatibilidade terá efeito direto e negativo sobre a resistência dos professores ao M-learning. $\operatorname{sim}$

H4: Experimentabilidade terá efeito direto e negativo sobre a resistência dos professores ao M-learning. não

H5: Observabilidade terá efeito direto e negativo sobre a resistência dos professores ao M-learning. não

Hipótese relacionando suporte institucional à resistência dos professores ao M-learning

H6: Suporte institucional terá efeito direto e negativo sobre a resistência dos professores ao M-learning. $\operatorname{sim}$

\begin{tabular}{|c|c|}
\hline \multicolumn{2}{|l|}{$\begin{array}{l}\text { Hipóteses relacionando os construtos da teoria da difusão de inovações à atitude geral de adoção do } \\
\text { M-learning }\end{array}$} \\
\hline $\begin{array}{l}\text { H7: Vantagem relativa terá efeito direto e positivo sobre a atitude de adoção dos professores ao } \\
\text { M-learning. }\end{array}$ & $\operatorname{sim}$ \\
\hline H8: Complexidade terá efeito direto e negativo sobre a atitude de adoção dos professores ao M-learning. & não \\
\hline H9: Compatibilidade terá efeito direto e positivo sobre a atitude de adoção dos professores ao M-learning. & $\operatorname{sim}$ \\
\hline $\begin{array}{l}\text { H10: Experimentabilidade terá efeito direto e positivo sobre a atitude de adoção dos professores } \\
\text { ao M-learning. }\end{array}$ & não \\
\hline H11: Observabilidade terá efeito direto e positivo sobre a atitude de adoção dos professores ao M-learning. & não \\
\hline \multicolumn{2}{|l|}{ Hipótese relacionando suporte institucional à atitude de adoção dos professores do M-learning. } \\
\hline $\begin{array}{l}\text { H12: Suporte institucional terá efeito direto e positivo sobre a atitude de adoção dos professores } \\
\text { ao M-learning. }\end{array}$ & $\operatorname{sim}$ \\
\hline \multicolumn{2}{|l|}{ Hipóteses relacionadas à atitude de adoção dos professores do M-learning. } \\
\hline $\begin{array}{l}\text { H13: Resistência dos professores ao M-learning terá efeito negativo sobre a atitude de adoção dos profes- } \\
\text { sores ao M-learning. }\end{array}$ & $\operatorname{sim}$ \\
\hline $\begin{array}{l}\text { H14: A atitude dos professores com relação ao uso do M-learning terá efeito positivo sobre a intenção do } \\
\text { comportamento de uso (adoção) dos professores ao M-learning. }\end{array}$ & $\operatorname{sim}$ \\
\hline
\end{tabular}

Fonte: Elaborado pelos autores 
na atitude geral de adoção dos professores ao M-learning. No que se refere à observabilidade, os resultados foram consistentes com os estudos de Duan, He, Feng et al. (20I0), com parte dos estudos de Lee, Hsieh e Hsu (20I I) e divergentes de Rogers (2003) e Machado, Bellinic e Leite (20/2). Sobre complexidade, a pesquisa chegou aos mesmos resultados de Duan, He, Feng et al. (20I0) e Lee, Hsieh e Hsu (20II), enquanto Machado, Bellinic e Leite (20I2) não encontraram relação significativa entre compatibilidade e atitude em relação à plataforma de e-learning. Entretanto, em relação à resistência dos professores ao M-learning, verificou-se como mediadora dos construtos observabilidade e atitude geral de adoção dos professores ao M-learning e também dos construtos complexidade e atitude geral de adoção dos professores ao M-learning.

A análise sugere que a percepção dos professores de que seu desempenho profissional melhora com o uso do M-learning, em comparação com seu desempenho sem esse uso, as relações que os professores fizerem com outras metodologias de ensino com o uso da tecnologia e o envolvimento das instituições de ensino com a preparação e a implementação do M-learning podem favorecer atitudes positivas de adoção dos professores dessa modalidade de ensino.

Experimentabilidade não mostrou efeito na resistência dos professores ao M-learning $(\mathrm{H} 4)$, nem na atitude de adoção dos professores ao M-learning ( $\mathrm{HIO}$ ), mesmo resultado obtido por Machado, Bellinic e Leite (20I2), contrariando a previsão de Rogers (2003). Duan, He, Feng et al. (2010) e Lee, Hsieh e Hsu (20II) encontraram efeito negativo da experimentabilidade na intenção de uso do e-learning e na utilidade percebida do e-learning respectivamente. Uma razão para este achado pode estar no fato de que professores não terem oportunidades efetivas de experimentar - M-learning antes de decidirem pelo seu uso no trabalho.

\section{CONSIDERAÇÕES FINAIS}

A principal contribuição teórica deste trabalho se deve ao fato de avaliar a resistência no processo de decisão de adoção de inovações, pouco estudada na literatura. Particularmente, pesquisas sobre a perspectiva dos professores são incipientes e dispersas, conforme apontaram Sánchez -Prieto, Olmos-Migueláñez e García-Peñalvo (2016). Os resultados fornecem indícios da influência significativa da resistência dos professores na atitude geral de adoção ao M-learning, o que se mostra consistente com Norzaidi, Chong e Salwani (2008) e Sanford e Oh (20I0), assim como indicou efeitos da atitude geral na intenção de adoção ao M-learning pelos professores.

Os resultados fornecem importantes insights e sugestões para a prática profissional para professores, IES, empresas e órgãos internacionais (ONU, OECD, Unesco) envolvidos com a Educação. Aos professores, a pesquisa pode ser um estímulo para a reflexão daqueles que gostariam de trazer inovações ao seu trabalho, mas não o fazem e nem mesmo sabem por que não o fazem. Buscar conhecer as suas possibilidades e vantagens, fazer uso do apoio da instituição de ensino quando houver, por exemplo, pode ajudar a reduzir a resistência ao M-learning e favorecer a atitude e a adoção por parte dos professores.

Do ponto de vista das IES, sugere-se o planejamento de iniciativas com os pro- 
fessores que explorem os atributos centrais do M-learning tais como portabilidade, mobilidade, experiência personalizada de aprendizagem, conforme apontado por diversos pesquisadores como Sharples, Taylor eVavoula (2007) e Kukulska-Hulme, Sharples, Milrad et al. (20II) para proporcionar a percepção de que o M-learning pode trazer desempenho melhor para 0 processo de ensino, ou seja, aumentando as percepções de vantagem relativa dos professores sobre o M-learning. $O$ planejamento de programas de capacitação, instrução e treinamento para facilitar o aprendizado, o conhecimento e a utilização dos professores do M-learning são ações que podem contribuir para reduzir a complexidade, na tentativa de reduzir a resistência ao M-learning.

Além do mais, a fim de proporcionar uma maior percepção de compatibilidade, os resultados orientam para ações que favoreçam a associação dos professores ao M-learning com metodologias consistentes com as experiências dos docentes no uso de tecnologia no ensino (exemplo: e-learning, plataforma da universidade, uso de vídeos, áudios no computador, Dropbox etc.) e com seu domínio da tecnologia móvel (tirar fotografias com o celular, filmar, gravar entrevistas). Essas experiências, se compatíveis com o quadro de referência dos professores, poderão reduzir a sua resistência ao M-learning.

Sobre a observabilidade, sugerem-se iniciativas para os professores que já usam o M-learning possam compartilhar suas práticas, seus resultados, demonstrando, assim, a eficácia do M-learning aos professores que não o utilizam com o objetivo de gerar oportunidades de observação dessa modalidade de ensino.

Como limitações do estudo, a amostra foi não probabilística e os questionários foram veiculados eletronicamente, ou seja, os respondentes são aqueles que se sentem minimamente confortáveis em responder pesquisas on-line. Questionários em papel poderiam tentar reduzir esse risco. Por essas razões, os resultados não podem ser generalizados para qualquer professor do ensino superior.

Outra possível limitação foi a possibilidade de os professores não conhecerem suficientemente o M-learning para responderem ao questionário. $O$ texto introdutório no cabeçalho do questionário e a reordenação das questões sugerida pelo pré-teste tiveram o objetivo de esclarecer a definição de M-learning e suas aplicações.

Para pesquisas futuras, sugere-se uma investigação mais profunda no construto observabilidade, em razão do efeito positivo entre observabilidade e resistência dos professores ao M-learning, contrário ao previsto a partir da literatura. 


\section{REFERÊNCIAS}

AJZEN, I. From intentions to actions: a theory of planned behavior. In: KUHL, J.; BECKMAN, J. (Eds.). Action control: from cognition to behavior. New York: Springer-Verlag, p. I I-39, 1985.

AJZEN, I.The theory of planned behavior. Organizational Behavior and Human Decision Processes, v.50, n.2, p. I79-2। I, I99।.

AKOUR, $\mathrm{H}$. Determinants of mobile acceptance: an empirical investigation in higher education. (Order No. 3408682, Oklahoma State University). ProQuest Dissertations and Theses, 379, 2010.

AL-EMRAN, M.; ELSHERIF, H.; SHAALAN, K. Investigating attitudes towards the use of mobile learning in higher education. Computers in Human Behavior, v. 56, Issue C, p. 93-102, 2016.

ANDERSON, J.; GERBING, D. Structural equation modeling in practice: $A$ review and recommended two-step approach. Psychological Bulletin, v. I03, n. 3, 4I I-23, 1998.

BYRNE, B. (20I0). Structural Equation Modeling with AMOS: Basic Concepts, Applications and Programming. 2nd ed. Routledge, NY.

CHIYANGWA, T.; ALEXANDER, P. Rapidly co-evolving technology adoption and diffusion models. Telematics and Informatics, v. 33, n. I, p. 56-76, 2016.

CHURCHILL, D.; CHURCHILL, N. Educational affordances of PDAs: A study of a teacher's exploration of this technology, Computers \& Education, v. 50, n. 4, p. |439-|450, 2008.

DAVIS, F. Perceived usefulness, perceived ease of use, and user acceptance of information technology. MIS Quarterly, v. 13, n. 3, p. 3 19339, 1989.

DAVIS, F; BAGOZZI, R.; WARSHAW, P. User acceptance of computer technology: a comparison of two theoretical models. Management Science, v. 35, n. 8, p. 982-I003, 1989.

DUAN,Y.; HE, Q.; FENG,W.; LI, D.; FU, Z. A study on e- take-up intention from an innovation adoption perspective: a case in China. Computers \& Education, v. 55, n. I, p. 237-246, 2010.

ELLEN, P.; BEARDEN, W.; SHARMA, S. Resistance to technological innovations: an examination of the role of self-efficacy and performance satisfaction. Journal of the Academy of Marketing Science, v. 19, n. 4, p. 297-307, 199I.

FERREIRA, J.; KLEIN, A.; FREITAS, A.; SCHLEMMER, E. Mobile learning: definition, uses and challenges. In: Wankel, L. A.; Blessinger, P. (Eds.). Increasing student engagement and retention using mobile applications: smartphones, skype and texting technologies. Cutting-edge Technologies in Higher Education, v.6, Emerald Group Publishing Limited,. p. 47-82, 2013.

FISHBEIN, M.; AJZEN, I. Belief, attitude, intention and behavior: an introduction to theory and research. Reading, MA: Addison-Wesley Publishing, 1975.

FORNELL, C.; LARCKER, D. Evaluating Structural Equation Models with Unobservable Variables and Measurement Error. Journal of Marketing Research, v. 18, n. I, p. 39-50, I98I.

HAIR, J.; BLACK, W.; BABIN, B.; ANDERSON, R. Multivariate Data Analysis. 7.ed. Upper Saddle River: Prentice-Hall, 2009.

HASHIM, K.; TAN, F.; RASHID, A. Adult learners' intention to adopt mobile learning: A motivational perspective. British Journal of Educational Technology, v. 46, n. 2, p. 38I-390, 2015.

HEIDENREICH, S.; KRAEMER, T. Passive innovation resistance:The curse of innovation? Investigating consequences for innovative consumer behavior. Journal of Economic Psychology, v. 5I, p. I34-I5I, 20 I5.

HINKIN, T. A review of scale development practices in the study of organizations. Journal of Management, v. 2I, n .5, p. 967-988, 1995.

HU, L.; BENTLER, P. Cutoff criteria for fit indexes in covariance structure analysis: Conventional criteria versus new alternatives. Structural Equation Modeling, v. 6, n. I, p. I-55, 1999.

HWANG, G.; WU, P. Applications, impacts and trends of mobile technology-enhanced learning: a review of 2008-2012 publications in selected $\mathrm{SSCl}$ journals. International Journal of Mobile Learning and Organisation, v. 8, n. 2, p. 83-95, 2014.

IGBARIA, M. End-user computing effectiveness: a structural equation model. Omega International Journal of Management Science, v. 18, n. 6, p. 637-652, 1990.

IGBARIA, M. User acceptance of microcomputer technology: an empirical-test. Omega International Journal of Management Science, v. 2I, n. I, p. 73-90, 1993.

JENKINS, H. Cultura da convergência. São Paulo:Aleph, 2008.

JOSHI, K. A model of users' perspective on change: the case of information systems technology implementation. MIS Quarterly,. v. I5, n. 2, p. 229-242, I99।.

KAPOOR, K.; DWIVEDI, Y., \& WILLIAMS, M. Innovation adoption attributes: a review and synthesis of research findings. European Journal of Innovation Management, v. I7, n. 3, p. 327-348, 2014.

KHADDAGE, F.; CHRISTENSEN, R.; LAI, W.; KNEZEK, G.; NORRIS, C.; SOLOWAY, E. A model driven framework to address challenges 
in a mobile learning environment. Education and Information Technologies, v. 20, n. 4, p. I-I6, 2015.

KUKULSKA-HULME, A.; SHARPLES, M.; MILRAD, M.;ARNEDILLO-SANCHEZ, I.;VAVOULA, G. The genesis and development of mobile learning in Europe. In D. Parsons (Ed.), Combining E-learning and M-learning: New Applications of Blended Educational Resources. Hershey, PA: IGI Global, p. I5I-I77, 20I I.

KURTZ, R.; PINA, F.; FERREIRA, J.; SILVA, J.; FREITAS, A.; GIOVANNINI, C. Adoção de M-learning por professores no ensino superior: uma investigação sobre aspectos favoráveis e possíveis barreiras. In: ENCONTRO NACIONAL DA ANPAD, 38, 2014. Rio de Janeiro,Anais... Rio de Janeiro, RJ:ANPAD, 2014.

KURTZ, R.; MACEDO-SOARES, T.; FERREIRA, J.; FREITAS, A.; SILVA, J. Fatores de Impacto na Atitude e na Intenção de Uso do M-learning: Um Teste Empírico. REAd. Revista Eletrônica de Administração, v. 2 I, n. I, p. 27-56, 2015.

LAPOINTE, L.; RIVARD, S. A multilevel model of resistance to information technology implementation. MIS Quarterly, v. 29, n. 3, p. 46I-486, 2005.

LEE, Y.; HSIEH, Y.; HSU, C. Adding innovation diffusion theory to the technology acceptance model: Supporting employees' intentions to use E-learning systems. Educational Technology \& Society, v. I4, n. 4, p. I24- I37, 20 I I.

LU,Y.; ZHOU, T.;WANG, B. Exploring Chinese users' acceptance of instant messaging using the theory of planned behavior, the technology acceptance model, and the flow theory. Computers in Human Behavior, v. 25, n. I, p. 29-39, 2009.

LUND, A. Measuring usability with the USE questionnaire. Usability Interface, v. 8, n. 2, p. 3-6, 200 I.
MA, L.; LEE, C.; GOH, D. Understanding news sharing in social media: An explanation from the diffusion of innovations theory. Online Information Review, v. 38, n. 5, p. 598-6I5, 2014.

MACHADO, P.; BELLINIC. G.; LEITE, J.Adoção de inovação tecnológica em educação a distância. Gestão \& Planejamento, v. I3, n. 2, p. 295 $300,2012$.

MARAKAS, G.; HORNIK, S. Passive Resistance Misuse: Overt Support and Covert Recalcitrance in IS Implementation. European Journal of Information Systems, v. 5, n. 3, p. 208-220, 1996.

MARTINKO, M.; HENRY, J.; ZMUD, R. An Attributional Explanation of Individual Resistance to the Introduction of Information Technologies in the Workplace. Behaviour \& Information Technology, v. I5, n. 5, p. 313-330, 1996.

MOORE, G.; BENBASAT, I. Development of an instrument to measure the perceptions of adopting an information technology innovation. Information Systems Research, v. 3, n. 2, p. 192-222, 1991.

MOTIWALLA, L. Mobile: a framework and evaluation. Computers \& Education, v. 49, n. 3, p. 58I-596, 2007.

NORZAIDI, M.; CHONG, S.; SALWANI, M. Perceived resistance, user resistance and managers' performance in the Malaysian port industry. In: ASLIB, 2008 Proceedings. Emerald Group Publishing Limited, v. 60, n .3, p. 242-264, 2008.

ONG, C.; LAI, J. Gender differences in perceptions and relationships among dominants of e-learning acceptance. Computers in Human Behavior,v. 22, n. 5, p. 816-829, 2006. ORLIKOWSKI,W.; GASH, D. Technological frames: Making sense of information technology in organizations. ACM Transactions on Information Systems, v. 12, n. 2, p. 174-207, 1994.
PARASURAMAN, A.; GREWAL, D.; KRISHNAN, R. Marketing Research, (2nd ed.). South-Western College Pub, 2006.

PINA, F;; KURTZ, R.; FREITAS,A.; SILVA, J.; RAMOS, F.A adoção do mobile learning: um estudo comparativo entre professores adotantes e não adotantes. In: ENCONTRO NACIONAL DAANPAD, 39, 20I5. Rio de Janeiro,Anais... Rio de Janeiro, RJ: ANPAD, 2015.

PODSAKOFF, P.; ORGAN D. Self-reports in organizational research: problems and prospects. Journal of Management, v. 2, n. 4, p. 53I-544, 1986.

ROGERS, E. Diffusion of innovations. New York:The Free Press, 2003.

SÁNCHEZ-PRIETO, J., OLMOSMIGUELÁÑEZ, S., \& GARCÍA -PEÑALVO, F. (20I6). Informal tools in formal contexts: Development of a model to assess the acceptance of mobile technologies among teachers. Computers in Human Behavior, 55, 519-528.

SANFORD, C.; OH, H.The role of user resistance in the adoption of a mobile data service. Cyberpsychology, Behavior, and Social Networking, v. I3, n. 6, p. 663-672, 2010.

SHARPLES, M.; TAYLOR, J.; VAVOULA, G. A theory of learning for the mobile age. In:ANDREWS, R.; HAYTHORNTHWAITE, C. (Eds.). The sage handbook of e research. London: Sage, p. 22 I-47, 2007.

SPERBER,A.Translation and validation of study instrument for cross-cultural research. Gastroenterology, v. I26, n. I, p. I24-I 28, 2004.

VENKATESH,V.; MORRIS, M.; DAVIS, G.; DAVIS, F. User acceptance of information technology: toward a unified view. MIS Quarterly, v. 27, n. 3, p. 425-478, 2003.

ZALTMAN, G.; DUNCAN, R. Strategies for planned change. New York: John Willey and Sons, 1977. 